\title{
Detection of griseofulvin in a marine strain of Penicillium waksmanii by ion trap mass spectrometry
}

\author{
K. E. Petit ${ }^{a}$, F. Mondeguer ${ }^{b}$, M. F. Roquebert ${ }^{c}$, J. F. Biard ${ }^{a}$ and Y. F. Pouchus ${ }^{a_{*}}$
}

\footnotetext{
a SMAB (Substances Marines à Activité Biologique), Université de Nantes, ISOMer, Faculté de Pharmacie, BP 53 508, 44035, Nantes cedex 01, France

${ }^{\mathrm{b}}$ IFREMER-DEL/PN, 1 rue de l'île d'Yeu, BP 21105, 44 311, Nantes Cedex 3, France

c Laboratoire de Cryptogamie, Muséum National d'Histoire Naturelle, 12 rue Buffon, 75 005, Paris, France

* fpouchus@sante.univ-nantes.fr Tel.: +33-2-40-41-28-70; fax: +33-2-40-41-28-58.
}

\begin{abstract}
A marine strain of Penicillium waksmanii Zaleski was isolated from a sample of seawater from shellfish-farming area in the Loire estuary (France). The in vitro marine culture showed an important antifungal activity. Bioassay-guided fractionation was used to purify the crude extract. Dereplication by electrospray-ion trap/mass spectrometry (ESI-IT/MS) afforded the identification of the antifungal compound, after a semi-purification consisting of two stages. A comparison of the ionic composition between the active and the non-active fractions allowed the detection of a monocharged ion at $\mathrm{m} / \mathrm{z} 353$ containing a chlorine atom, which could be attributed to the antifungal griseofulvin $[\mathrm{C} 17 \mathrm{H} 17 \mathrm{ClO} 6+\mathrm{H}]+$. Multi-stage fragmentation $(\mathrm{MSn})$ confirmed the identity of the $\mathrm{m} / \mathrm{z} 353$ ion of the antifungal fraction as griseofulvin. It is the first description of griseofulvin production by a strain of $P$. waksmanii and the first chemical study of a strain of this species isolated from marine temperate cold water.
\end{abstract}

Keywords: Penicillium waksmanii; Marine fungi; Antifungal; Griseofulvin; Mass spectrometry ESIIT/MS 


\section{Introduction}

During our ongoing search for fungal occurrence in shellfish-farming areas, more than 400 strains had been isolated from marine sediment, shellfish or sea water (Sallenave et al., 2000). One of them, identified as Penicillium waksmanii Zaleski, inhibited the growth of moulds in its vicinity. Marine fungi have proved to be a rich source of new bioactive natural products (Cuomo et al., 1995; Liberra and Lindequist, 1995; Farooq Biabani and Laatsch, 1998; Jensen and Fenical, 2000). Because of their particular living conditions (salinity, nutrition, higher pressure, temperature variations, competition with bacteria, viruses and other fungi), they may have developed specific secondary metabolic pathways compared with terrestrial fungi (Liberra and Lindequist, 1995). It was thus settled to isolate and identify the causal metabolite.

$P$. waksmanii Zaleski is an ubiquitous anamorphic fungus, widespread at once in land (Pitt, 1979), in guano of swallow (Doria and Guglielminetti, 1997), in mosquitoes (Costa et al., 1998, Costa and Oliveira, 1998) and in various aquatic environments such as sewage sludge (Ismail et al., 1994) or algae (Dewey et al., 1983; Amagata et al., 1998).

Actually, different metabolites have been isolated from cultures of $P$. waksmanii: two alkaloids (Kozlovskii et al., 1997), four pyrones (pyrenocines A, B, D and E) with significant cytotoxicity on P388 lymphocytes $(<1.5 \mu \mathrm{g} / \mathrm{ml})$ and three sulfur-containing dioxopiperazines (Amagata et al., 1998) but no antifungal metabolites has already been described.

This paper describes the first study of a strain of $P$. waksmanii isolated from temperate cold water, which exhibits a new biological activity for this species.

\section{Materials and methods}

\subsection{Fungal strain}

The studied strain has been isolated from a sample of seawater collected in a shellfishfarming area from the Loire estuary (France). Fungal strain was identified according to Pitt's method as Penicillium waksmanii Zaleski. Pitt's method is based on microscopic characters and colony morphology of the isolated strain, cultured on three standard media (Pitt, 1979).

The strain has been registered into the National Museum of Natural History culture collection (Paris, France) under the reference number LCP 99.43.43. 


\subsection{Solid phase culture}

Cultures were performed in $500 \mathrm{ml}$ glass flasks containing $50 \mathrm{ml}$ of a solid medium composed of 0.5 g peptone, 1.75 g glucose and 0.75 g agar (Becton Dickinson, Sparks, USA), dissolved in natural filtered seawater. The medium was inoculated with $P$. waksmanii conidia taken from stock cultures (stored at $-18^{\circ} \mathrm{C}$ ) with a platinum loop. Each flask was then incubated for 14 days at $27^{\circ} \mathrm{C}$ in natural light.

\subsection{Warm Solid/Liquid Extraction WSLE}

Cultures were stopped by warming on a hot plate $\left(60^{\circ} \mathrm{C}\right)$ to allow the agar to melt and were then extracted with warm ethyl acetate. After shaking, suspension was left at room temperature until the resolidification of the agar phase. The liquid organic phase was then removed, was sterilely filtered under vacuum $(0.45 \mu \mathrm{m}$ PTFE membrane filters, Sartorius, Göttingen, Germany) and was evaporated to obtain a crude extract.

\subsection{Antifungal assay}

Antifungal activity was detected by the paper disk diffusion method according to Hadacek and Greger (2000). A pathogen isolate of Candida albicans was used as test organism. Amphotericin B (Calbiochem, Meudon, France) was used as a positive control (10 $\mu \mathrm{g} / \mathrm{ml}$ in methylene chloride with $10 \%$ dimethyl sulfoxyde).

Test plates (8 cm diameter) were prepared with Sabouraud agar (Becton Dickinson, Sparks, USA) in distilled water (1.3 g for $20 \mathrm{ml}$ ) and inoculated with a suspension of C. albicans $\left(10^{6}\right.$ cells $\left./ \mathrm{ml}\right)$. For testing, extracts $(2.5 \mathrm{mg})$ were dissolved in $1 \mathrm{ml}$ methylene chloride. Paper disks (9 mm diameter, Prolabo Merck Eurolab, Gradignan, France) were impregnated with 20 - $\mu$ l aliquots of extracts or control. After evaporating the organic solvent, paper disks were disposed on plates (one test extract and one positive control). Plates were incubated for $24 \mathrm{~h}$ at $37^{\circ} \mathrm{C}$ and the growth inhibition zone was detected. All samples were tested in triplicate. 


\subsection{Semi-purification}

Vacuum Liquid Chromatography (VLC) was performed using silica gel $60 \AA$ 35$70 \mu \mathrm{m}$ as solid phase (SDS, Peypin, France). Elution was successively realised with hexane/methylene chloride 50:50 (v/v), methylene chloride/ethyl acetate 99:1 (v/v), 80:20 (v/v) and ethyl acetate $100 \%$. For separating $100 \mathrm{mg}$ of crude extract, $10 \mathrm{~g}$ of silica and $100 \mathrm{ml}$ of each eluant were used.

High-performance liquid chromatography (HPLC) was performed on an analytical reverse column (Intersphere $C_{18} 5 \mu \mathrm{m}, 250$ x 4.6 mm, Interchim, Montluçon, France). Mobile phase (methanol/water 85:15 v/v) was delivered by a 305-Pump (Gilson, France) at a constant flow rate of $0.5 \mathrm{ml} / \mathrm{min}$. Samples were injected as pure methanolic solutions $(10 \mathrm{mg} / \mathrm{ml})$ in a Rheodyne valve (1-ml sample loop). Volumes of $500 \mu$ l were injected by a $500-\mu l$ syringe (Hamilton, Reno, NV, USA). Detection was performed at $254 \mathrm{~nm}$ using a 115-UV model detector (Gilson) coupled to a SE-120 model integrator (BBC Guerz Metrawatt).

Each solvent (ethyl acetate, methylene chloride, hexane and methanol) was of Normapur quality (SDS, Peypin, France) and distilled prior to use. Water was purified by a Milli-Q system (Millipore, Bedford, MA, USA).

\subsection{Identification by mass spectrometry (MS) analyses}

MS analyses were carried out using an electrospray ionization-ion trap (ESI-IT) mass spectrometer (LCQ Finnigan, Atlanta, GA, USA). Mass analyses were conducted in positive mode with initial source parameters as shown in Table 1. All spectra were acquired and analysed by LCQ Xcalibur software (Finnigan, Atlanta, GA, USA). Total current ion mass spectra (Fullscan mode) were acquired between $\mathrm{m} / \mathrm{z} 80$ and $\mathrm{m} / \mathrm{z} 1000$. Charge state and isotopic distribution were analysed by a narrow-scan range mode (Zoomscan mode) and compared with isotopic profile calculation performed with Isopro 3.0 MS/MS software (Internet freeware: see http://members.aol.com/msmssoft/). An isolation mass width of $m / z=1$ was applied during $M^{n}{ }^{n}$ analyses of parent ions. Scanning was recorded for about 0.3 min corresponding to 12 scans. Spectra were acquired in profile mode with a boxcar smoothing of 7 points.

Concerning MS analysis, fungal extracts and pure griseofulvin solutions were prepared at different concentrations (100 $\mu \mathrm{g} / \mathrm{ml}$ and $10 \mu \mathrm{g} / \mathrm{ml}$ respectively) in a mixture of methanol/water 95:5 (v/v) with $0.1 \%$ of trifluoroacetic acid. These solutions were infused 
directly into the ESI probe with a $250-\mu \mathrm{l}$ automated syringe (Hamilton, Reno, NV, USA) at a flow-rate of $3 \mu \mathrm{l} / \mathrm{min}$.

Methanol of HPLC quality grade was purchased from Baker (Deventer, Holland). Water was purified by a Milli-Q system (Millipore, Bedford, MA, USA). Griseofulvin was obtained from Sigma-Aldrich (St.Quentin Fallavier, France).

Table 1: Comparison of the initial source parameters of the ESI-IT/MS with the optimised parameters for the analysis of the $m / z 353$ ion

\begin{tabular}{|c|c|c|}
\hline Parameters & Initial source parameters & Optimised parameters \\
\hline source voltage & $4.5 \mathrm{kV}$ & $60 \mathrm{kV}$ \\
\hline $\begin{array}{c}\text { sheath gas flow rate (nitrogen, arbitrary } \\
\text { units) }\end{array}$ & 60 & 0 \\
\hline $\begin{array}{c}\text { auxiliary gas flow rate (nitrogen, } \\
\text { arbitrary units) }\end{array}$ & 0 & $160^{\circ} \mathrm{C}$ \\
\hline capillary temperature & $160^{\circ} \mathrm{C}$ & $10 \mathrm{~V}$ \\
\hline capillary voltage & $19 \mathrm{~V}$ & $5 \mathrm{~V}$ \\
\hline tube lens & $0 \mathrm{~V}$ & $-2 \mathrm{~V}$ \\
\hline octopole 1 voltage & $-2.5 \mathrm{~V}$ & $-6 \mathrm{~V}$ \\
\hline octopole 2 voltage & $-7 \mathrm{~V}$ & $-16 \mathrm{~V}$ \\
\hline interoctopol lens & $-16 \mathrm{~V}$ & 6 \\
\hline
\end{tabular}

\section{Results}

\subsection{Semi-purification}

Semi-purification of fungal cultures was bioassay-guided through different chromatographic techniques. Extraction of $3.65 \mathrm{l}$ peptone/sea water cultures was performed using the WSLE protocol. It gave $742 \mathrm{mg}$ of crude extract. This extract was divided into 100mg aliquots and subjected to VLC separation on silica-phase. The whole antifungal activity was detected in the methylene chloride/ethyl acetate 80:20 (v/v) fraction. An average of $9 \mathrm{mg}$ of this fraction was obtained for $100 \mathrm{mg}$ of crude extract.

Repeated injections of the previous active fraction were performed on a HPLC reverse-phase column. The antifungal activity was found within the 6-to-8-min fraction. After evaporation, the active fraction yielded $5.8 \mathrm{mg}$ of a yellow and oily mixture. 


\subsection{ESI/IT/mass analysis}

Among the five fractions collected by HPLC, only one showed an antifungal activity. We first analysed the ionic composition of the active and non-active fractions by ESI-IT/MS and then compared them. Although the Fullscan mass spectra were still very complex (Fig. 1), a dominant monocharged peak at $\mathrm{m} / \mathrm{z} 353$ could be detected as the main difference between the active fraction and the others. The isotopic distribution of this peak (Zoomscan analysis, Fig. 2) revealed the presence of one chlorine atom.

Figure 1: Fullscan mass spectrum of the HPLC active fraction of $P$. waksmanii

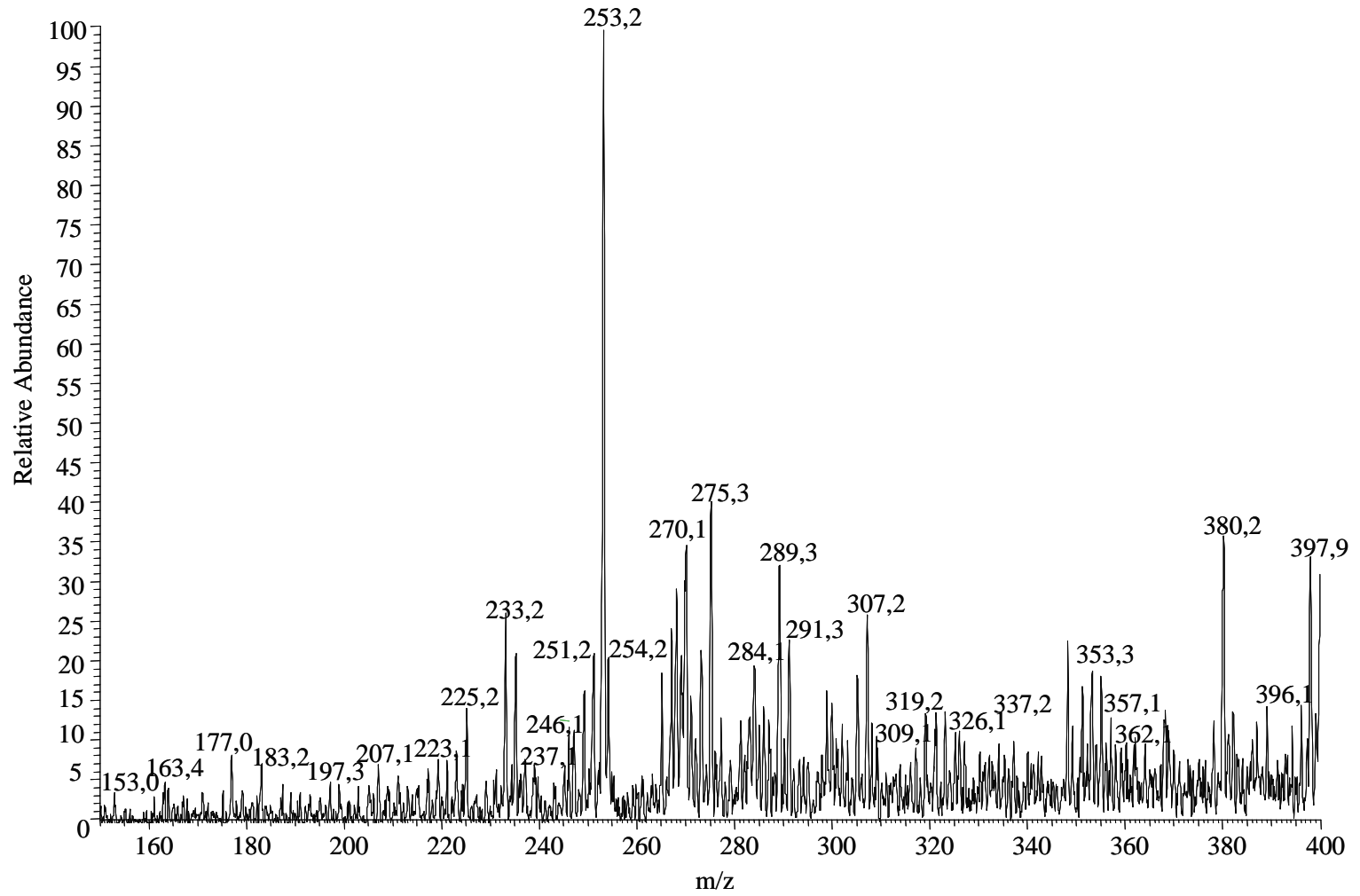

Figure 2: Isotopic distribution spectrum in positive mode of peak of $\mathrm{m} / \mathrm{z} 353$ in the HPLC active fraction of P. waksmanii

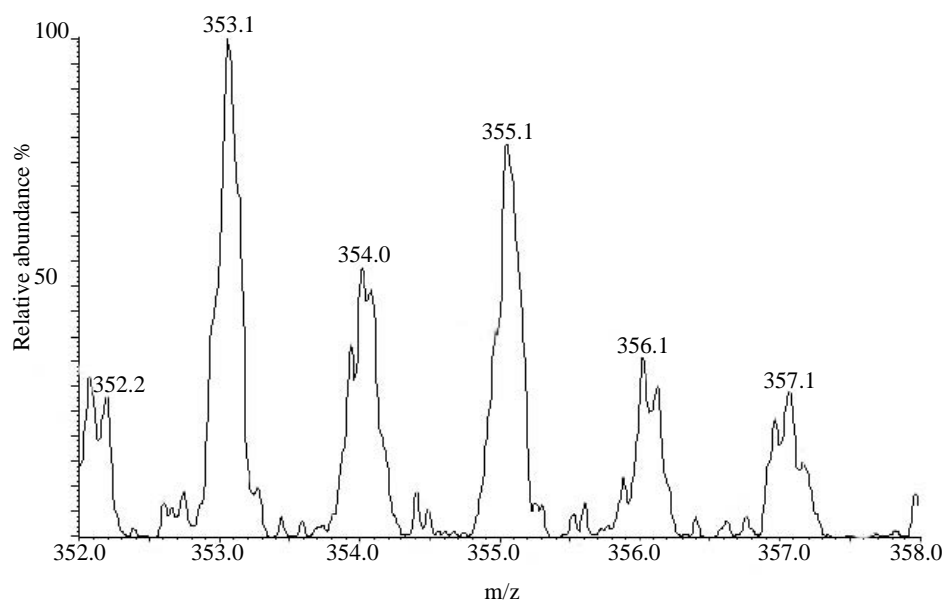


3.3 Identification of griseofulvin by $M^{n}$ analysis

Since griseofulvin (Fig. 3) presents the same molecular weight ( $\mathrm{MW}=352 \mathrm{Da}$ ), contains a chlorine atom and has an antifungal activity, a comparison of the obtained peak and pure griseofulvin has been performed.

A pure griseofulvin solution $(10 \mu \mathrm{g} / \mathrm{ml})$ was directly injected into the ESI-IT/MS. Fullscan MS showed a major peak at $\mathrm{m} / \mathrm{z} 353$ corresponding to the protonated griseofulvin $[\mathrm{M}+\mathrm{H}]^{+}$with the same isotopic distribution compared to the observed unknown 353 peak. ESI parameters were automatically optimised to obtain the best signal for this ion (Table 1).

Collision energy of $27 \%$ was required for MS/MS analysis of griseofulvin. A major chlorinated product ion appeared at $m / z 215$ associated with three less intense ions at $m / z 321$ (chlorinated, relative abundance 26\%), 285 (chlorinated, 70\%) and 165 (non-chlorinated, $45 \%$ ) (Fig. 4a). In the same conditions, the unidentified $\mathrm{m} / \mathrm{z} 353$ ion gave the same product ions (Fig. 4b) confirming that this peak could be attributed to griseofulvin.

Figure 3: Chemical structure of griseofulvin

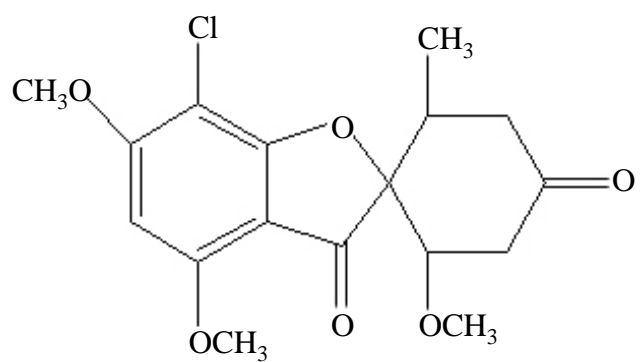


Figure 4: MS/MS spectra in positive mode of

a) Griseofulvin control m/z 353

b) Peak of $m / z 353$ in the active fraction of $P$. waksmanii

a)

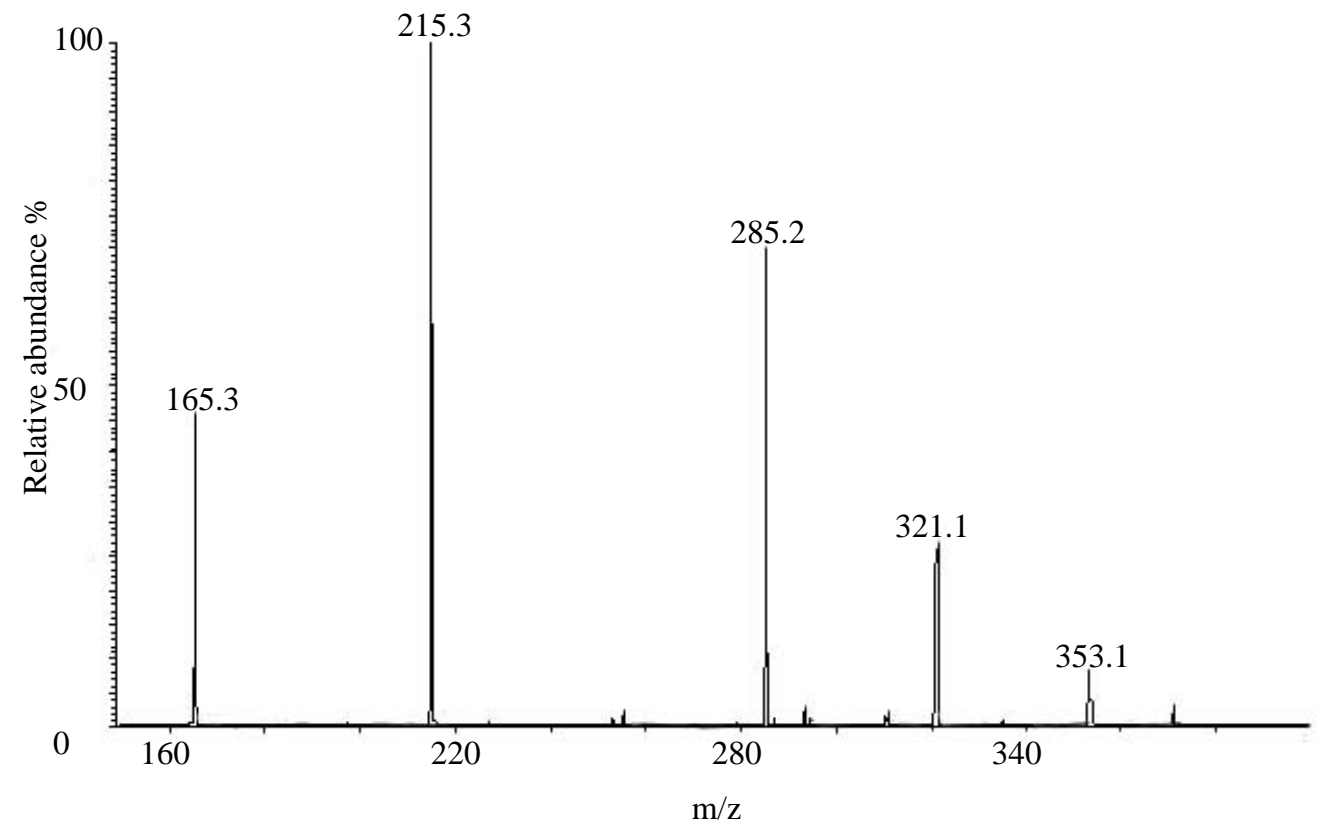

b)

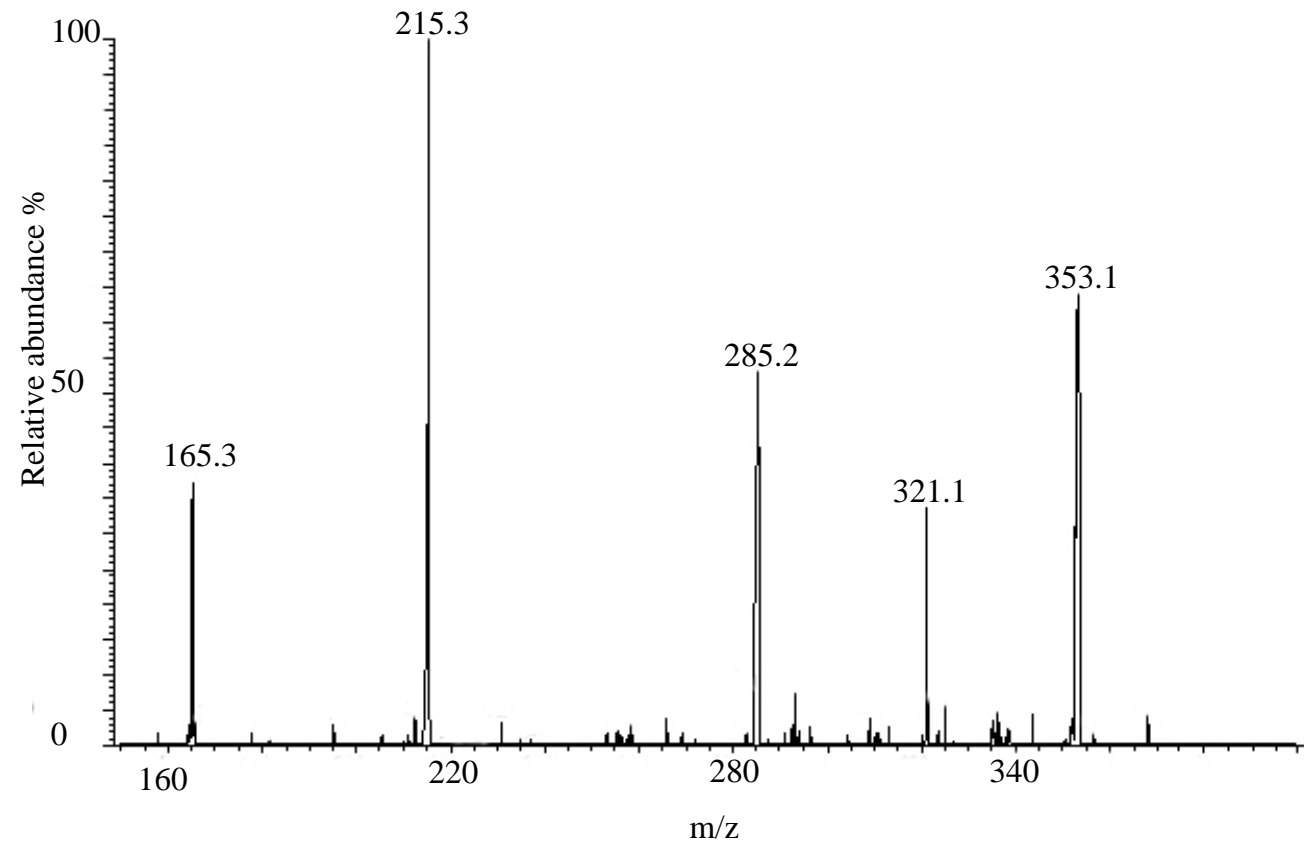




\section{Discussion}

This work was aimed to study marine fungi as a new source of bioactive compounds. The original warm solid/liquid extraction (WSLE) protocol, developed in this study, seems to be a powerful technique for fungal compounds extraction from solid culture medium: it is rapid, effective and it allows extraction of excreted metabolites as well as mycelial compounds.

MS/MS is a powerful technique allowing identification of compounds in a complex extract with a high degree of certainty, which could not be obtained with classical methods such as TLC or HPLC-UV (Fischer and Riegelman, 1966; Zia et al., 1980; Frisvad and Filtenborg, 1989; Venkatadasu et al., 2000). Our study is a typical application of the use of ESI/MS providing "mass profiles" of fungal extracts (Smedsgaard and Frisvad, 1996). Then, after fractionation of the crude extract guided by an antifungal bioassay, ESI-IT/MS/MS allowed the identification of griseofulvin as an active principle of $P$. waksmanii.

Griseofulvin is a well-known antifungal compound which had already been isolated from four terrestrial species of Penicillium (Oxford et al., 1939; Brian, 1949; Araujo et al., 1990) and from a non-identified marine species (Namikoshi et al., 2000). Therefore we report here for the first time the presence of griseofulvin in the metabolic content of $P$. waksmanii.

It is not the first antifungal metabolite isolated from a marine fungus (Liu et al., 2002; Schlingmann et al., 2002), indicating that marine-adapted strains could synthesize those bioactive compounds in marine culture conditions. Studying marine fungal metabolites is relevant both for the pharmaceutical industry, as they are varied and can be highly bioactive, and for the understanding of their ecological role. The reason why bioactive compounds are product is not known, but it is assumed that many of them act as chemical defence. As a result, antifungal metabolites biosynthesis could be induced in response to other fungal competitors, thus providing a selective advantage for their development in natural marine environment. For instance, it has been reported that the production of the antibiotic pestalone by a marine fungal strain of Pestalotia sp. was effective only when this strain was co-cultured with a marine bacterium (Cueto et al., 2001).

Our study brings here new evidences of the potential development of filamentous saprophytic fungi in the sea, although this hypothesis is still discussed by several authors. Moreover, the studied strain did not grow on a solid medium prepared with distilled water, indicating that it required salt in the medium for its development and thus it is well-adapted to marine environment. 


\section{Acknowledgments}

This paper was part of the $\mathrm{PhD}$ thesis of KE Petit and supported financially by the Regional Council of Pays de la Loire (France). 


\section{References}

Amagata, T., Minoura, K., Numata, A., 1998. Cytotoxic metabolites produced by a fungal strain from a Sargassum algae. J. Antibiot. 51, 432-434.

Araujo, O.E., Flowers, F.P., King, M.K., 1990. Griseofulvin: a new look at an old drug. DICP, The Annals of Pharmacotherapy 24, 851-854.

Brian, P.W., 1949. Studies on the biological activity of griseofulvin. Ann. Bot. - London, N.S. XIII (49), 59-77.

Costa, G.L., Moraes, A.M.L., Oliveira, P.C., 1998. Pathogenic action of Penicillium species on mosquito vectors of human tropical diseases. J. Basic Microb. 38 (5-6), 337-341.

Costa, G.L., Oliveira, P.C., 1998. Penicillium species in mosquitoes from two Brazilian regions. J. Basic Microb. 38 (5-6), 343-347.

Cueto, M., Jensen, P.R., Kauffman, C., Fenical, W., Lobkovsky, E., Clardy, J., 2001. Pestalone, a new antibiotic produced by a marine fungus in response to bacterial challenge. $\mathrm{J}$. Nat. Prod. 64 (11), 1444-1446.

Cuomo, V., Palomba, I., Perretti, A., Guerriero, A., D'Ambrosio, M., Pietra, F., 1995. Antimicrobial activities from marine fungi. J. Mar. Biotechnol. 2, 199-204.

Dewey, F.M., Donelly, K.A., Foster, D., 1983. Penicillium waksmanii isolated from a red seaweed, Eucheuma striatum. Trans. Br. Mycol. Soc. 81 (2), 433-434.

Doria, M., Guglielminetti, M., 1997. Micromiceti associati ai nidi di Rondine commune (Hirundo rustica L.). Mic. Ital. 3, 95-99.

Farooq Biabani, M.A., Laatsch, H., 1998. Advances in chemical studies on low-molecular weight metabolites of marine fungi. J. Prak. Chem. - Chem. Ztg. 340, 589-607. 
Fischer, L.J., Riegelman, S., 1966. Quantitative determination of griseofulvin and griseofulvin-4'-alcohol in plasma by fluorimetry on thin layer chromatograms. J. Chromatography A 21, 268-274.

Frisvad, J.C., Filtenborg, O., 1989. Terverticillate penicillia: chemotaxonomy and mycotoxin production. Mycologia 81, 837-861.

Hadacek, F., Greger, H., 2000. Testing of antifungal natural products: methodologies, comparability of results and assay choice. Phytochem. Analysis 11, 137-147.

Ismail, M.A., Omar, S.A., 1994. Factors affecting ureolytic activities of Penicillium waksmanii isolated from sewage sludge. Folia Microbiol. 39 (5), 441-444.

Jensen, P.R., Fenical, W., 2000. Marine microorganisms and drug discovery: current status and future potential. In Drugs from the sea, Basel, Karger, eds Fusetani N., 6-29.

Kozlovskii, A.G., Vinokurova, N.G., Zhelifonova, V.P., Ozerskaya, S.M., 1997. Alkaloid formation by Penicillia of the series Fellutana and Canescentia (in Russian). Mikrobiologiia 66 (4), 514-519.

Liberra, K., Lindequist, U., 1995. Marine fungi - A prolific resource of biologically active natural products ? Pharmazie 50 (H9), 583-588.

Liu, C.H., Meng, J.C., Zou, W.X., Huang, L.L., Tang, H.Q., Tan, R.X., 2002. Antifungal metabolite with a new carbon skeleton from Keissleriella sp. YS4108, a marine filamentous fungus. Planta Med. 68 (4), 363-365.

Namikoshi, M., Kobayashi, H., Yoshimoto, T., Meguro, S., Akano, K., 2000. Isolation and characterization of bioactive metabolites from marine-derived filamentous fungi collected from tropical and sub-tropical coral reefs. Chem. Pharm. Bull. 48 (10), 1452-1457.

Oxford, A.E., Raistrick, H., Simonart, P., 1939. Griseofulvin, $\mathrm{C}_{17} \mathrm{H}_{17} \mathrm{O}_{6} \mathrm{Cl}$, a metabolic product of Penicillium griseofulvum Dierckx. Biochem. J. 33, 240-248. 
Pitt, J.I., 1979. The genus Penicillium and its teleomorphic states Eupenicillium and Talaromyces. Academic Press, London.

Sallenave-Namont, C., Pouchus, Y.F., Bardouil, M., Lassus, P., Roquebert, M.F., Verbist, J.F., 2000. Toxigenic saprophytic fungi in marine shellfish farming areas. Mycopathologia 149, 21-25.

Schlingmann, G., Milne, L., Carter, G.T., 2002. Isolation and identification of antifungal polyesters from the marine fungus Hypoxylon oceanicum LL-15G256. Tetrahedron 58: 68256835.

Smedsgaard, J., Frisvad, J.C., 1996. Using direct electrospray mass spectrometry in taxonomy and secondary metabolite profiling of crude fungal extracts. J. Microbiol. Methods 25, 5-17.

Venkatadasu, V., Muralidhar, R.V., Panda, T., 2000. Analytical techniques for griseofulvin. Bioprocess Eng. 22 (3), 201-204.

Zia, H., Proveaux, W.J., O'Donnell, J.P., Ma, J.K.H., 1980. Chromatographic analysis of griseofulvin and metabolites in biological fluids. J. Chromatography Biomedical App. 181, 77-84. 